\title{
Further delineation of the G syndrome: a manageable genetic cause of infantile dysphagia
}

\author{
GOLDER N WILSON* AND WILLIAM J OLIVER † \\ From *the Department of Pediatrics, The Montreal Children's Hospital, McGill University, Montreal, \\ Quebec, Canada; and the Department of Pediatrics, University of Michigan, Ann Arbor, Michigan, USA.
}

SUMmaRY Three families including five subjects with the $\mathrm{G}$ or Opitz-Frias syndrome are added to 23 published cases who had dysphagia; characteristics of the two affected relatives were added to 19 well documented published reports. The data from index cases support the concept of the $G$ syndrome as a constellation of midline defects, which include hypertelorism or telecanthus $(89 \%)$, oesophageal dysmotility $(69 \%)$, laryngotracheal clefts $(44 \%)$, cleft palate or bifid uvula $(34 \%)$, heart defects $(29 \%)$, hypospadias $(100 \%$ of males), renal or ureteral anomalies $(42 \%)$, and mental retardation (38\%). Affected relatives, often identified by hypertelorism, dysphagia, or hypospadias, had a much lower incidence of associated defects and mental retardation. They provide a more rounded but still biased view of a syndrome compatible with normal intelligence and life span. The data do not support a highly characteristic face in the $G$ syndrome, which discriminates it from the phenotypically similar BBB syndrome. The variable expressivity and five cases of male to male transmission observed in 18 families are consistent with autosomal dominant inheritance. Vigilance for the morphological characteristics of $G$ syndrome in patients with dysphagia is underscored by the potential for normal development with appropriate intervention.

Since Illingworth's categorisation' ${ }^{\prime}$ of infantile dysphagia into structural or neuromuscular causes, numerous examples of clefts or of palsies affecting the gastrointestinal tract have been defined. A malformation syndrome combining problems in both categories was first described in 1969 by Opitz et $a l^{2}$ in four patients from the $G$ family. Subsequent publications $^{3-6}$ added more patients from the $G$ family and a second $\mathrm{J}$ family, defining hypertelorism, laryngotracheo-oesophageal clefts, oesophageal dysmotility, and hypospadias as cardinal features of the $G$ syndrome. Paediatric consideration of the $G$ syndrome in the evaluation of infantile dysphagia is important, since oesophageal function improves with age and appropriate measures to prevent aspiration can result in normal lifespan and intelligence. Diagnosis of the syndrome also allows genetic counselling with anticipatory management of pregnancy and delivery.

At the time of this review, 26 children with the $G$ or Opitz-Frias syndrome from 16 families have been reported. ${ }^{2-18}$ three of whom did not have the

This study was presented in part at the David W Smith Symposium. Burlington. Vermont. August 1986.

Received for publication 13 November 1986.

Revised version aceepted for publication 26 February 1987. cardinal feature of dysphagia. ${ }^{3}{ }^{13}$ The predominance of affected males ( 23 cases) may in part reflect ascertainment bias due to hypospadias, and several cases of male to male transmission ${ }^{13}{ }^{16-18}$ suggest autosomal dominant inheritance. The existence of mildly affected family members is consistent with variable expressivity and implies that the syndrome may be much more common than the number of reported cases would suggest. In addition, the expanded spectrum of the $G$ syndrome as seen in mildly affected patients makes its phenotypic separation from related conditions such as the BBB or hypertelorism-hypospadias syndrome ${ }^{12} 1.3$ less clear. To clarify these issues further, we report an additional five cases of the $G$ syndrome in three families, and consider the pattern of anomalies in mildly affected family members. An approach to infants with dysphagia which considers the expanded phenotype of the $G$ syndrome is discussed.

\section{Case reports}

CASE 1

This six year old white female (fig $1 \mathrm{~b}$ ) weighed $2640 \mathrm{~g}$ at birth; the 34 week gestation was complicated by polyhydramnios and premature rupture of mem- 


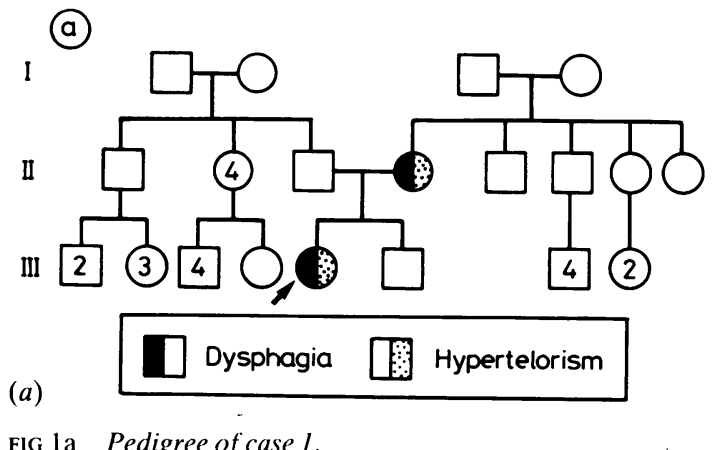

FIG la Pedigree of case 1 .

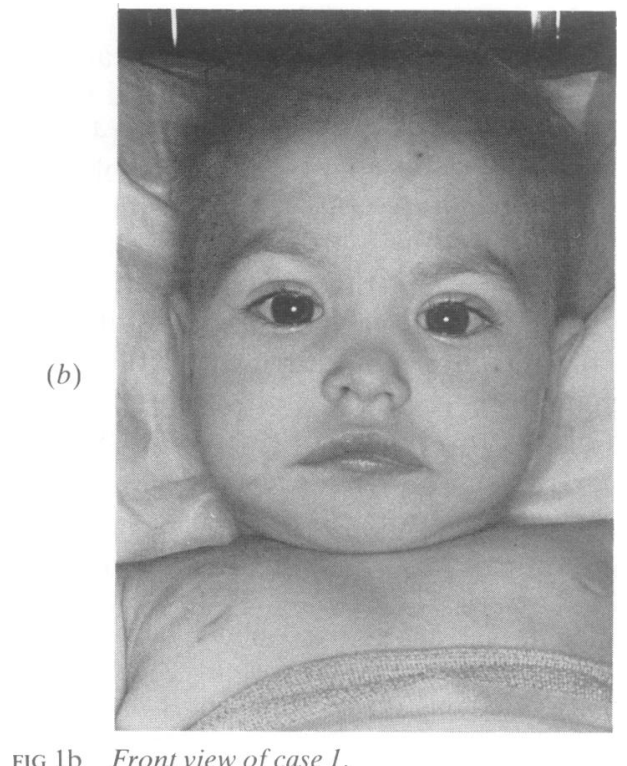

FIG 1b Front view of case 1.

branes. Following an uncomplicated vertex vaginal delivery and normal nursery stay, the child developed bloody stools and was readmitted to hospital at 13 days of age. Physical findings included hepatomegaly ( $5 \mathrm{~cm}$ below costal margin), hypotonia with poor head control, and decreased spontaneous movement. Laboratory evaluations for sepsis, infectious diarrhoea, congenital infection, and metabolic disease were normal, but the child required hyperalimentation and broad spectrum antibiotics before achieving normal nutrition on Pregestamil ${ }^{\circledR}$.

At two months of age, the child was admitted for treatment of cough, conjunctivitis, and lethargy: a chest $x$ ray showed right middle lobe and diffuse interstitial infiltrates. The liver was $1 \mathrm{~cm}$ below the costal margin and a normal sweat sodium level was $\stackrel{+}{\rightarrow}$ obtained. The child improved rapidly with erythromycin therapy, but required many admissionso to hospital during her first year for suspected pneumonia, failure to thrive, and developmental $\frac{\bar{c}}{2}$ delay. At the age of one year, extensive evaluation $\vec{\alpha}$ disclosed the following findings: height $69 \mathrm{~cm}(5$ th centile), weight $6.72 \mathrm{~kg}$ (below the 3rd centile), head circumference $44.5 \mathrm{~cm}$ (25th centile), anterior. fontanelle open $(1 \times 1 \mathrm{~cm})$, haemangiomas over the $\overrightarrow{\vec{\omega}}$ left flank and left inner thigh, telecanthus $(3 \cdot 1 \mathrm{~cm}, \stackrel{\omega}{\omega}$ 90th centile) with hypertelorism $(5 \cdot 2 \mathrm{~cm}, 95$ th centile), shallow nasal bridge, midline clefts in the hard palate (posterior $1 \mathrm{~cm}$ ), cleft epiglottis, bifid $\mathrm{N}$ uvula, liver $4 \mathrm{~cm}$ below the costal margin, and $\omega$ general hypotonia with increased lower extremity $\vec{f}$ reflexes $(3+)$. Laboratory findings included incoordination of the soft palate without evidence of gastro-oesophageal reflex (barium swallow), left $\vec{z}$ upper lobe cavitation and presumed lung abscess (chest $x$ ray and bronchoscopy), normal culture $\frac{\hat{O}}{\alpha}$ results and blood chemistries except for Staphylococcus aureus from the nares, normal liver function $\varnothing_{\infty}$ tests including coagulation times and $\alpha_{1}$ antitrypsin, normal serum immunoglobulins, complement, haemagglutinin, candida reactivity, auditory evoked response, serum amino acids, thyroid, and lactate, and an EEG showing diffuse slowing without paroxysmal features. The child responded to $\stackrel{\mathbb{Q}}{\circ}$ aggressive pulmonary therapy and multiple anti- $\underset{\vec{P}}{\vec{F}}$ biotics and was discharged on Enfamil formula. 웅

Multiple admissions for respiratory infection con- $\bar{T}$ tinued and the child was in hospital from the age of 16 months to 20 months for resection of the left upper lobe cavitation. A Hill-Nissen fundoplication $\overline{3}$ and gastrostomy tube placement were performed for $\dot{-}$ gastro-oesophageal reflux and oesophageal incoor- 3 . dination which were now apparent on barium $\delta$ swallow. The diagnosis of $\mathrm{G}$ syndrome was made and the child monitored for reflux by serial barium $\mathrm{O}$ studies. Despite the absence of observable reflux after operation, the subsequent course included recurrent pneumonias from staphylococcus, pseudomonas, and candida, persistent left pneumatoceles, $ه$ chronic otitis externa and interna, multiple staphylo- $N$ coccal cutaneous abscesses, pseudomembranous ్ㅗ colitis from Clostridium difficile, failure to thrive, alopecia, and developmental delay. The gastros-

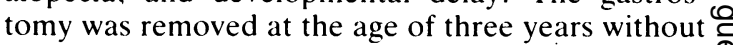
subsequent reflux. Immune evaluation has shown $\stackrel{\mathscr{\Phi}}{\rightarrow}$ normal $\mathrm{T}$ cell mitogens, neutrophil function, and staphylococcal killing. Turbinate biopsy suggested 0 hypoactive cilia. Mild developmental delay may $\overrightarrow{\mathbb{D}}$ reflect over 50 hospital admissions during her six $\underset{\mathbb{D}}{\stackrel{\circ}{\circ}}$ years. A family history (fig la) showed that the mother, who has mild hypertelorism and a high? 
palate, required tube feeding because of swallowing difficulties as a child.

CASE 2

This three month old white male (fig $2 a$, b) weighed $3 \mathrm{~kg}$ following an uncomplicated term gestation and vaginal vertex delivery. The mother had two healthy children by a previous marriage and there was no history of dysphagia, hypospadias, or other birth defects in the family. Severe cyanosis and respiratory distress after delivery prompted evaluation which revealed tetralogy of Fallot with severe pulmonic stenosis, hypoplastic pulmonic arteries, and right sided aortic arch as documented by echocardiography. Laboratory findings included normal EEG, cranial ultrasound, and pneumogram, severe oesophageal dysmotility with delayed gastric emptying and gastro-oesophageal reflux, normal testosterone response to HCG, and a 46.XY normal male karyotype. The clinical course included severe failure to thrive, developmental delay, and chronic cardiopulmonary disease despite a left BlalockTaussig shunt, Nissen fundoplication, and gastrostomy. The patient died at the age of 10 months after cardiorespiratory arrest due to probable but undocumented sepsis. Necropsy findings included a length of $61 \mathrm{~cm}$ (50)th centile), weight $3.9 \mathrm{~kg}$ (50th centile), head circumference $42 \mathrm{~cm}$ ( 50 th centile),

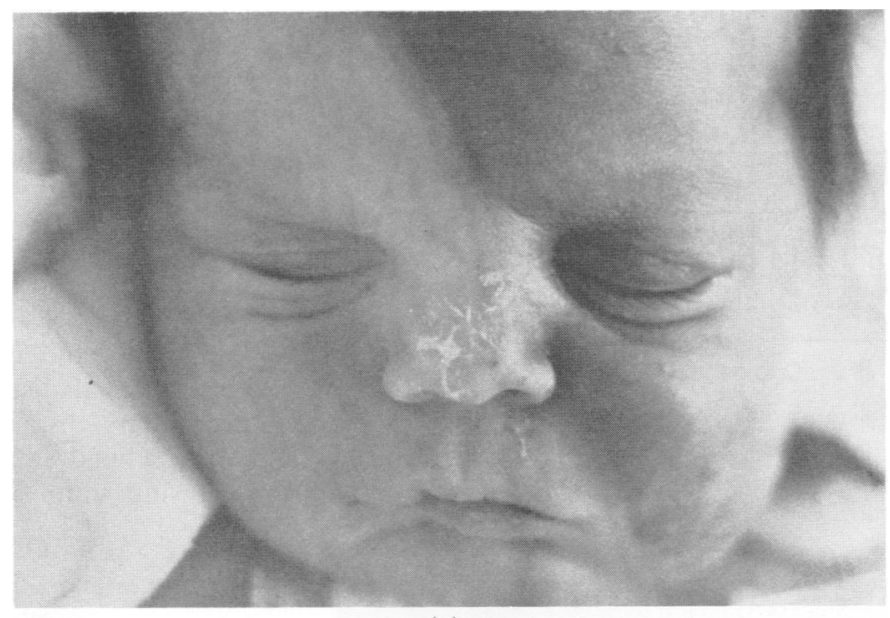

(a)

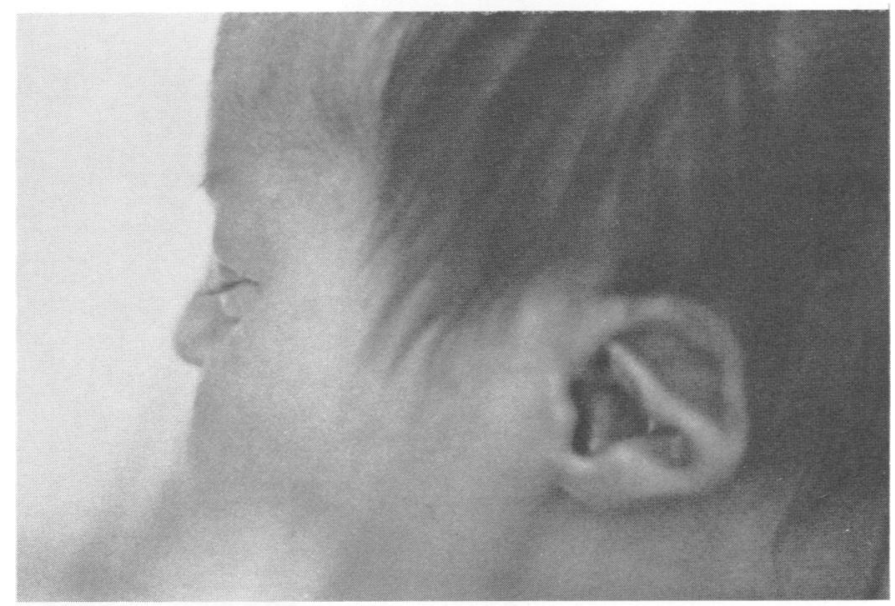

(b)

FIG 2 Frontal (a) and lateral (b) view of case 2. 
relative hypertelorism for a four month old with an interpupillary distance of $4.5 \mathrm{~cm}$ (50)th centile for nine months), prominent forehead, prominent nasal bridge, low set ears, right choanal atresia, short neck, bilateral fifth finger clinodactyly, bilateral simian creases, seven of 10 arch dermatoglyphic patterns, ambiguous genitalia with small penis $(1 \mathrm{~cm})$, mild hypospadias, bilateral cryptorchidism, horseshoe kidney, marked underdevelopment of the white matter of the cerebral hemispheres and descending systems of the brain stem, bilateral rudimentary intra-abdominal testicles, normal cerebellum, and normal gallbladder. Brain histology showed reduced white matter throughout the cerebral hemispheres, cerebral peduncles, and cortico-

(a)
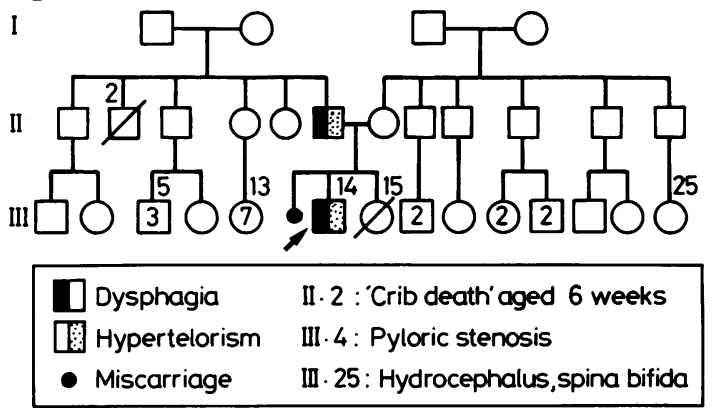

Fig 3a Pedigree of case 3 . spinal tracts. There was no evidence of a demyeli- $\underset{\vec{\sim}}{\vec{*}}$ nisation process. The olfactory bulbs and tracts $\stackrel{\vec{\rho}}{\rightarrow}$ could not be recognised. There were normal ganglion cells in the oesophagus.

CASE 3

This 13 month old white male (fig $3 b$ ) weighed $2980 \mathrm{~g}$ at birth after a 36 week gestation complicated by hypertension and eclampsia. The mother was 24.0 years old with a previous eight week miscarriage. $\overrightarrow{\vec{\omega}}$ After caesarean section with Apgar scores of 9 and $\stackrel{\mathscr{\rho}}{\omega}$ 9. cyanosis and tachypnoea were noted at the age of $\overline{\overline{3}}$ one hour and an oesophageal tube could not be passed. Tracheo-oesophageal fistula and oesophageal of atresia were corrected surgically at five days and a feeding gastrostomy placed until two months of age. $\vec{v}$ Two episodes of aspiration pneumonitis led to 0 recognition of oesophageal stenosis, oesophageal $\frac{0}{\partial}$ incoordination, and gastro-oesophageal reflux: a $\overrightarrow{\vec{z}}$ Nissen fundoplication with gastrostomy was performed at three and a half months. Dilatation of the oesophagus was performed and the patient has fed $\vec{P}$ orally since the age of five months with a single $\mathscr{\infty}$ episode of severe aspiration occurring at 11 months.

Physical findings included a height of $79 \mathrm{~cm}(80)$ th centile), weight $11.3 \mathrm{~kg}$ (80)th centile), head circumference $48.5 \mathrm{~cm}$ ( 80 th centile), shallow nasal bridge with intercanthal distance of $2.2 \mathrm{~cm}(80) \mathrm{th} \approx$ centile), interpupillary distance $4.5 \mathrm{~cm}(90$ th cen- $\stackrel{\square}{\triangle}$ tile), prominent forehead, highly arched palate, thin $\vec{F}$ upper lip, small and simplified but normally posi- $\frac{0}{3}$

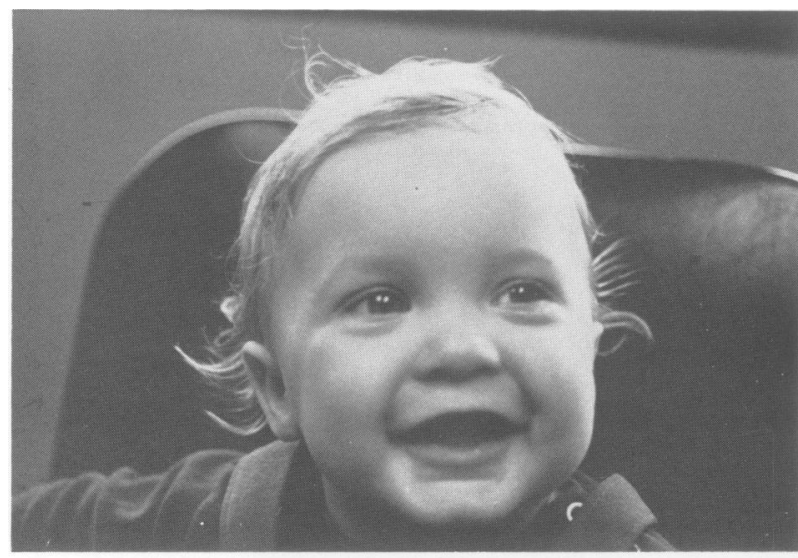

(b)

FIG 3b, c Frontal view of case 3(b) and his father (c) aged II.

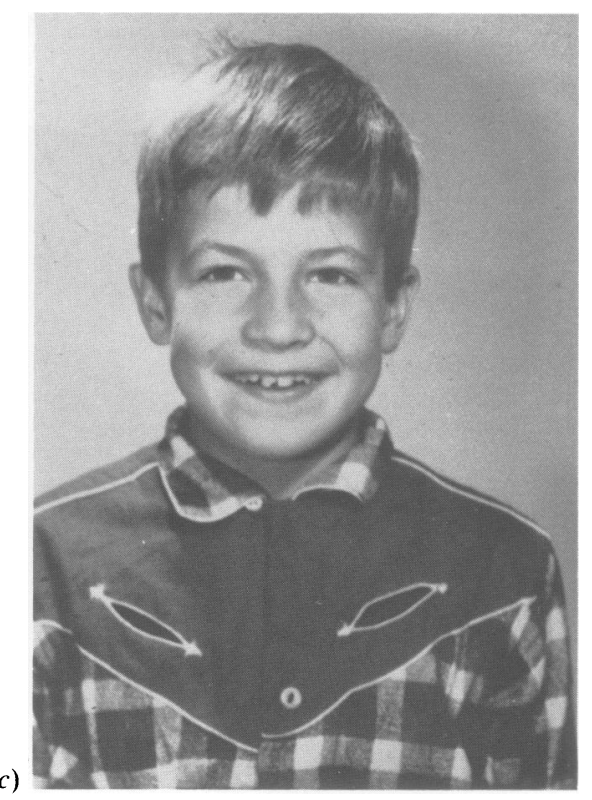

(c) 
tioned ears, normal palmar creases, mild hypospadias, and normal intellectual development. Echocardiography had identified a patent ductus arteriosus at the age of three months without left to right shunting.

A family history (fig 3a) showed that the father (fig 3c) had feeding problems with an immature oesophagus' during his youth. His formula was switched several times because of vomiting and persistent diarrhoea. As an adult he still gags easily and chokes on large pieces of food. His height is 180 $\mathrm{cm}$ ( 75 th centile), weight $102 \mathrm{~kg}$ (97th centile), head circumference $59.5 \mathrm{~cm}$ (97th centile), interpupillary distance $6 \mathrm{~cm}$ (97th centile), intercanthal distance $3.1 \mathrm{~cm}$ (75th centile), and outer canthal distance 9 $\mathrm{cm}$ ( 75 th centile). He has a highly arched palate but no hypospadias. As shown in the pedigree, a paternal brother died a 'crib death' at six weeks and a paternal nephew had surgically corrected pyloric stenosis. The couple has subsequently had a stillborn female fetus at 32 weeks' gestation after CorGard $^{\circledR}$ and Dyazide ${ }^{\circledR}$ treatment for hypertension. Fetal necropsy at the Teratology Unit, University of Michigan, revealed micrognathia, ques- tionable small ears, and tapered fingertips; the tissue was too autolysed for karyotype analysis. Placental examination revealed haemorrhagic endovasculitis.

\section{Results}

The clinical manifestations of our cases 1 to 3 are summarised in the table with other reported patients with the $G$ syndrome. The data from the definitive patients support a strong influence of the $G$ syndrome gene upon diverse systems including the face (hypertelorism or telecanthus in $89 \%$ ), respiratory tract (laryngotracheal clefts in $44 \%$, aspiration pneumonitis in $85 \%$ ). gastrointestinal tract (demonstrable oesophageal incoordination in $69 \%$ of those studied, swallowing difficulties in $81 \%$ ), genitourinary tract (hypospadias in $100 \%$ of males, renal or ureteral anomalies in $42 \%$ of patients studied), and heart (29\% assuming complete ascertainment). In addition to their typical clinical or family histories, our cases 1 to 3 each had more than six of the above nine features as evidence for the diagnosis of $G$ syndrome.

TABLE Manifestations of $G$ syndrome patients and their relatives.

\begin{tabular}{|c|c|c|c|c|c|c|c|}
\hline \multirow[t]{2}{*}{ Clinical manifestatioms } & \multirow{2}{*}{$\begin{array}{l}\text { P'uhlished } \\
\text { cases }\end{array}$} & \multicolumn{3}{|c|}{ Presem cases } & \multirow[t]{2}{*}{ Totul } & \multirow[t]{2}{*}{$\%$} & \multirow[t]{2}{*}{ Relutives } \\
\hline & & 1 & 2 & 3 & & & \\
\hline \multicolumn{8}{|l|}{ Giencral } \\
\hline Malc sex & $23 / 26$ & - & + & + & $25 / 29$ & 86 & $7 / 21$ \\
\hline Family history & $23 / 26$ & + & - & + & $25 / 29$ & 86 & \\
\hline Gestation & Mcan $3 x$ wk & 34 & 411 & 36 & $3 \times \cdot 5$ & & \\
\hline Birth weight & Mcan $2981 g$ & $2(x+1)$ & $3(60)$ & $29 \times 1$ & 2970 & & \\
\hline \multicolumn{8}{|l|}{ (raniofacial } \\
\hline Hypertelorism & $22 / 24$ & + & + & - & $24 / 27$ & 89 & $16 / 20$ \\
\hline Telecanthus & $\mid 11 / 11$ & - & - & - & $101 / 14$ & 71 & \\
\hline Oral cleft: & $9 / 26$ & + & - & - & $10 / 29)$ & .34 & \\
\hline Malformed cars & $15 / 17$ & - & + & + & $17 / 20$ & 85 & \\
\hline \multicolumn{8}{|l|}{ Gl/pulmonary } \\
\hline Dysphaigia & $19 / 24$ & + & + & + & $22 / 27$ & 81 & $5 / 8$ \\
\hline Pharyngeal clefts & $101 / 24$ & + & - & + & $12 / 27$ & +4 & \\
\hline Hoarse voice & $9 / 23$ & & + & & $10 / 24$ & 42 & \\
\hline Ocsophageal dysmotility & $8 / 13$ & + & + & + & $11 / 16$ & (1) & \\
\hline Tracheo-oesophageal fistula & $5 / 25$ & - & - & + & $6 \sqrt{2} 8$ & 21 & \\
\hline Gastrostomy & $5 / 21$ & + & + & + & $N 24$ & 33 & \\
\hline Aspiration pncumonia & $14 / 17$ & + & + & + & $17 / 20$ & 85 & $3 / 21$ \\
\hline \multicolumn{8}{|l|}{ (icnitourinary } \\
\hline Hypospatdias & $2.3 / 2.3$ & & + & + & $25 / 25$ & $I(x)$ & +17 \\
\hline (ryptorchidism & $5 / 2.3$ & & + & - & $6 / 25$ & 24 & \\
\hline Renal/urcteral defects & +111 & & + & - & $5 / 13$ & 38 & \\
\hline Imperforate anus & $3 / 25$ & - & - & - & $3 / 2 x$ & 11 & \\
\hline \multicolumn{8}{|l|}{ Miscelliancous } \\
\hline Cardiac & $6 / 25$ & - & + & + & $x / 2 x$ & 29) & \\
\hline Mental retardation & $6 / x$ & + & + & - & $N / 21$ & 38 & $1 / 21$ \\
\hline
\end{tabular}

*Numerator: number of patients affected. Denominator: number of patients living long enough to manifest feature and in whom feature specifically included or excluded.

tIncludes cleft plate, uvula, tonguc.

‡Includes cleft epiglottis. larynx. trachea, ocsophagus. 
The data do not support the existence of a highly characteristic facies for $\mathrm{G}$ syndrome patients as cited by Funderburk and Stewart ${ }^{13}$ as a means of distinguishing the condition from the BBB hypertelorismhypospadias syndrome. Thus, small or narrow palpebral fissures were found in three of nine patients for which the feature was mentioned, mongoloid slant to the eyes in three of 10 , antimongoloid slant to the eyes in four of 10 , prominent nasal bridge in five of 11 , shallow nasal bridge in six of 11 , anteverted nares in six of 12 , epicanthal folds in one of 12 , central groove to the nasal tip in two of nine, and prominent occiput in three of eight. A prominent forehead and parietal bossing were present in all eight patients for which the feature was mentioned. While several of the six of 25 patients with mental retardation might have sustained secondary damage because of severe dysphagia, the presence of EEG abnormalities and the severity of case 2 suggests a still undefined incidence of congenital neurological disease. The cerebral hypomyelinisation shown at necropsy in case 2 needs additional confirmation for this to be considered a part of the syndrome. A normal karyotype has been documented in 12 cases.

Approximately 21 relatives of the previously and presently reported $\mathrm{G}$ syndrome cases have been described in sufficient detail to provide an estimate of the incidence of cardinal features. The predominance of affected female relatives (14/21), the lower incidence of swallowing difficulties (5/8), hypertelorism (16/20), and hypospadias (4/7), and the presence of only one patient with mild mental retardation gives a less biased view of a syndrome compatible with a relatively normal face and life span. This additional evidence for variable expressivity and the five reported cases of male to male transmission now available provide strong support for autosomal dominant inheritance of the $G$ syndrome and necessitates revision of its listing ${ }^{19}$ as an $\mathrm{X}$ linked disorder.

\section{Discussion}

Opitz and Gilbert ${ }^{20}$ have discussed the vertebrate midline as a developmental field which seems particularly susceptible to morphogenetic variation. Defects of the $G$ syndrome which have midline origin include hypertelorism, widow's peak, bifurcated nasal tip, short lingual frenulum, clefts of the palate, tongue, uvula, larynx, trachea, and oesophagus, hypospadias, urethral duplication, bifid scrotum, and imperforate anus. Of interest in view of midline problems is the apparent immune defect in patient 1 , with recurrent otitis and sinusitis reminis- cent of Kartagener patients with situs inversus and immotile cilia. ${ }^{21}$

The dominance of midline problems in the $G O$ syndrome explains its phenotypic overlap ${ }^{12}$ with a $\frac{\bar{O}}{\bar{D}}$ related developmental field defect, the BBB or $\frac{\bar{c}}{\frac{1}{2}}$ telecanthus-hypospadias syndrome. While certain $\stackrel{\mathbb{Q}}{\square}$ families with $G$ syndrome have a face which is quite distinct from that of typical patients with the BBB syndrome, compilation of facial features does not $\stackrel{\circ}{\circ}$ support a definitive separation of these two $\overrightarrow{\vec{\omega}}$ conditions. ${ }^{13}$ Many $G$ syndrome patients will have $\stackrel{\mathscr{\rho}}{\omega}$ obvious hypertelorism with a flattened bridge of the $\overline{\overline{3}}$ nose, frontal and parietal bossing, prominent occiput, narrow palpebral fissures, anteverted nares, $\mathcal{G}$ malformed ears, and a highly arched or cleft $\omega$ palate. $^{23}$ BBB patients are more likely to have $\vec{G}$ cranial asymmetry, epicanthic folds, strabismus, and 0 a prominent nasal bridge without the prominent forehead or occiput or parietal eminences. ${ }^{12} 1322$

Perhaps more important than discrimination of related midline defect syndromes is an appreciation of the risks which these conditions confer upon $\overrightarrow{\overrightarrow{0}}$ neonatal feeding and development. Patients with $\mathscr{\infty}^{\circ}$ the $G$ syndrome may have diverse causes for ${ }^{\circ}$ dysphagia which range from oesophageal dysmotility to severe defects in closure of the embryonic laryngotracheal groove. ${ }^{4}$ These may be difficult to demonstrate even by special evaluation and the infant with hypertelorism, dysphagia, and particu- $\stackrel{\varnothing}{\complement}$ larly aspiration pneumonia should be regarded as a $\overrightarrow{\vec{P}}$ medical emergency until a safe means of feeding can $\frac{3}{3}$ be established. Urgent evaluations should include $\bar{\tau}$ direct laryngoscopy under general anaesthesia by an experienced endoscopist ${ }^{15}$ in addition to oesophagograms with a water soluble contrast media. ${ }^{3 \times}$ In addition to repair of laryngotracheal clefts, signifi- $\dot{\sigma}$ cant gastro-oesophageal reflux or oesophageal incoordination or both should prompt consideration 8 of a Nissen-Hill fundoplication with feeding gastrostomy. Since aspiration of saliva may still occur after 을 these measures in severely affected infants, cervical oesophagostomy should also be considered. Failure to institute timely management has severe and deadly results, as indicated by the nine of 26 infants $O$ in the table dying before the age of one year and the $N$ severe pulmonary problems in case 1 . The improve- N ment in oesophageal function with age ${ }^{\prime}$ and the $\sigma$ normal mentality of most $G$ syndrome patients provide strong incentives for aggressive manage- $\frac{C}{0}$ ment.

The information now available on 18 families with $\mathrm{G}$ syndrome is strongly suggestive of autosomal ${ }^{\circ}$ dominant inheritance with variable expressivity. $\overrightarrow{\mathbb{D}}$ The high number of affected males may reflect $\frac{\vec{D}}{\mathbb{D}}$ ascertainment bias due to hypospadias or greater $\frac{\varrho}{0}$ severity with increased abortion in affected females. 
The predominance of female relatives of $G$ syndrome cases as listed in the table would favour ascertainment bias as an explanation. The existence of apparently normal heterozygotes, along with the low rate (three of 26 cases at most) of spontaneous mutation, indicates both a higher incidence and lesser severity of the syndrome than reported cases would suggest. The latter fact should be considered during genetic counselling: even apparently normal couples, such as the parents of case 2, might have their pregnancies followed for fetal anomalies or polyhydramnios by ultrasound. Since as many as 59 to $82 \%$ of patients with tracheo-oesophageal fistula have gastro-oesophageal reflux after operative repair, ${ }^{2.3}$ more attention to the nature of laryngotracheal clefts and craniofacial features in this population may reveal a subset with the genetic and pulmonary risks characteristic of the $G$ syndrome.

\section{References}

' Illingworth RS. Sucking and swallowing difficultics in infancy: diagnostic problem of dysphagia. Arch Dis ('hild 1944:44: 655-65.

- Opitz JM. Frias JL. Gutenberger JE. Pellet JR. The G syndrome of multiple congenital anomalies. Birth Defects 1969:V(2):95-101.

' Little JR. Opitz JM. The G syndrome. Am J Dis ('hild 1971:121:505-7.

+ Gilbert EF, Viseskul C. Mossman HW. Opitz JM. The pathologic anatomy of the $G$ svndrome. $Z$ Kinderheilkd 1972:11:290-8.

5 Kasner J. Gilbert EF. Viseskul C. Deaon J. Herrmann JPR. Opitz JM. Studies of malformation syndromes. VID. The G syndrome: further observations. Z Kinderheilkd 1974:118:81-5.

" Arya S. Viseskul C. Gilbert EF. The G syndrome-additional observations. Ain J Med Genet 1980:5:321-4.

7 Coburn TP. G syndrome. Am J Dis (hild 1970):120:466.

* Van Biervliet JPGM. Van Hemel JO. Familiar occurrence of the $G$ syndrome. Clin Genet 1975:7:238-44.
"Frias JL. Rosenbloom AL. Two new cases of the G sundrome. Birth Defects 1975:XI(2):54-7.

11" Pederson IL. Mikkelsen M. Oster J. The G svndrome. A fourgeneration family study. Hum Hered 1976:26:66-71.

1 Miller PR. Pathak A. Decancq HG Jr. Hypertelorismhypospadias syndrome with laryngotracheoesophageal cleft. J Pediatr 1977:90:157-8.

I2 Cordero JF. Holmes LB. Phenotypic overlap of the BBB and G syndromes. Am J Med Genet 1978:2:145-52.

${ }^{13}$ Funderburk SJ, Stewart R. The G and BBB syndromes: case presentations, genetics, and nosology. Am J Med Genet 1978;2: $131-44$.

${ }^{14}$ Greenberg CR. Schraufnagel D. The G syndrome: a case report. Am J Med Genet 1979:3:59-64.

1.5 Coté GB. Katsantoni A. Papadakou-Lagovanni S. et al. The G syndrome of dysphagia. ocular hypertelorism and hypospadias. Clin Genet 1981:19:473-8.

16 Farndon PA. Donnai D. Male to male transmission of the $G$ syndrome. Clin Genet 1983:24:446-8.

17 Chemke J. Shor E. Ankori-Cohen H. Kazuni E. Male to male transmission of the G syndrome. Clin Genet 1984:26:164.

is Allanson JE. The G syndrome: an unusual family. David W Smith Symposium, Burlington, 1985. In: Proceedings of the Greenwood Genetic Center. Vol 5. Clinton, SC: Jacobs Press, 1986:168-9.

19 McKusick V. Mendelian inheritance in man. 6th ed. Baltimore: Johns Hopkins University Press. 1984.

2" Opitz JM. Gilbert EF. CNS anomalies and the midline as a 'developmental field'. Am J Med Genet 1982:12:443-55.

$\Rightarrow$ Afzelius AB. Kartagener`s syndrome and abnormal cilia. $N$ Engl J Med 1977:297:1011-4.

"2 Opitz JM. Smith DW. Summitt RL. Hypertelorism and hypospadias. A newly recognized hereditary malformation syndrome. J Pediatr 1965:67:968.

23 Jolley SG. Johnson DG. Roberts CC. et al. Patterns of gastroesophageal reflux in children following repair of esophageal atresia and distal tracheoesophageal fistula. J Pediatr Surg 1980):15:857-62.

Correspondence and requests for reprints to $\mathrm{Dr}$ Golder N Wilson, Department of Pediatrics, Suite 303 Medical Office Building. William Beaumont Hospital, 3535 W Thirteen Mile Road, Royal Oak, Michigan 48072, USA. 